\title{
Generalized Multiway Relay Network: Proposition and Effective Rate Analysis
}

\author{
Marcio Henrique Doniak, Richard Demo Souza, and Bartolomeu F. Uchôa-Filho
}

\begin{abstract}
A multiway relay network (mRN), which is widely employed in the literature, is extended to represent a larger number of practical scenarios, such as a vehicular ad hoc network (VANET). For the proposed system, generalized multiway relay network $(\mathrm{g}-\mathrm{mRN})$, three transmission strategies are presented and analyzed in terms of effective rate, for different numbers of users. Assuming that the average signal-to-noise ratio (SNR) is known to the transmitters, the maximum effective rate is obtained through the optimal choice of the message rates. We also derive the effective rate for fixed message rates. Numerical results show that the best strategy under the optimal message rates is the one in which all users transmit simultaneously in the multiple access phase. But when considering fixed message rates, the best transmission strategy depends upon the SNR. These results are important in that they can be used as guidelines for selecting the right strategy and message rates depending on the situation.
\end{abstract}

Index Terms-Multiway relay network, Effective rate, Message rate, VANET.

\section{INTRODUCTION}

$\mathbf{M}$ ULTIWAY Relay Networks (mRNs) have been receiving a great deal of attention recently. In a $\mathrm{mRN}$, users exchange information through the assistance of a relay node. Each user transmits its information to the relay, which in turn forwards this information to all users through the multiway relay channel (mRC). This network was first proposed by Günduz et al. in [1], and then extended and elaborated on by the same authors in [2]. More recently, the same network was studied in the works [3], [4], where it has been named $\mathrm{mRN}$, a terminology which has been used ever since.

The presence of a relay in the mRN brings several benefits, such as: improving the coordination of the transmissions, expanding the users reach, and providing greater energy efficiency and higher information transfer rate [2]. Several communication networks were studied under the mRN concept, such as wireless sensor networks (WSN) [2], satellite networks [5], and vehicular ad hoc networks (VANETs) [6].

In the original works of Günduz et al. (viz, [1], [2]), as well as in the subsequent ones (viz, [6], [7]), users would

M. H. Doniak is with the Area of Telecommunications, Campus São José, Federal Institute of Santa Catarina, São José - SC, 88103310, Brazil, e-mail: mdoniak@ifsc.edu.br.

R. D. Souza and B. F. Uchôa-Filho are with Department of Electrical and Electronics Engineering, Federal University of Santa Catarina, Florianópolis, SC, 88040900, Brazil, e-mail: richard.demo@ufsc.br, uchoa@eel.ufsc.br.

Manuscript received February 28, 2018; revised April 11, 2018; accepted May 8, 2018.

The authors are grateful for the financial support provided by $\mathrm{CNPq}$, Brazil. A preliminary version of this paper was presented in XXXV Simpósio Brasileiro de Telecomunicações (SBrT15), São Pedro, SP, Brazil, September 3-6, 2017.

Digital Object Identifier: 10.14209/jcis.2018.12 communicate only through the relay, i.e., no direct links existed between participating users. Herein, we remove this restriction and extend their channel model to allow direct communication within certain subsets of users. With this modification, several practical scenarios can be now represented. Before enumerating the contributions of the present work, we introduce some important related works.

\section{A. Prior Related Research}

In the system model considered in [1], [2], users are assumed to have full-duplex channel access to the relay, but no user can communicate directly with another. The main contribution of [2] is the derivation of fundamental limits in terms of the system throughput.

Similar system models were subsequently considered in [3] [6], [8]-[12], where different strategies for exchanging information among users were proposed and analyzed. They all consist of two phases: one in which users transmit, called multiple access phase (MAC), and the other where the relay broadcasts signals to all users, called the broadcast phase (BC). Users intend to send independent messages to all users, and all transmit at the same common transmission rate. In these strategies, at each time slot (TS) a subset of users and/or the relay may transmit simultaneously.

The transmission strategies proposed in [5], [8] and [9] consider that all users transmit simultaneously during the first TS. In [8] and [9], users can communicate not only with the relay but also with one another. While in [8] all the users are arranged to belong to a single cluster, like in the original work [2], in [9] several clusters are possible.

On one hand, in a given strategy the more users are scheduled to transmit their signals simultaneously during the MAC phase, the fewer time slots (TS) the strategy requires for the users to complete the exchange of information. However, on the other hand, more simultaneous transmissions imply higher signal processing complexity in the associated receivers. Therefore, to reduce this complexity in the MAC phase, some authors have recently restricted the number of users transmitting simultaneously to two [3], [10]-[12]. These works seek to optimize the performance/throughput of the system through the choice of the optimal pairs of users to transmit simultaneously and the optimal orderings.

Particularly interesting is the recent work [12], where several clusters, each one with its own relay station, are defined. In the system model, some clusters are responsible for the information exchange among the users of the cluster, while one cluster is elected responsible for the exchange of information 
among all the users of the network. So, there is a distinction between the information that stays in the cluster, called private information, and the one that is exchanged among clusters, called public information. All transmissions are performed in pairs.

A network with a massive number of users, like a VANET or a WSN, is propitious to use a mRN as a model. In this regard, the authors in [4] considered a highly dense network where some users are selected to transmit simultaneously through half-duplex channels. Similar to [8] and [9], users can communicate with one another and the received information from the other users is employed as side information to increase even further the number of users. In [6], users are allowed to transmit more than once, and the number of users transmitting simultaneously varies in each TS. There are no direct links between users. The relay uses the amplify-andforward technique to forward the received signal to all users. In a similar work [13], users can transmit alone or with other users. In the three works [4], [6], [13], users transmit in an uncoordinated fashion in order to avoid scheduling complexity.

\section{B. Motivation and Our Contribution}

The motivation for this work comes from the VANETs application, in which vehicles normally exchange information among themselves [14]-[16]. As an example of a VANET, Figure 1 shows an urban scenario where two vehicles, $\mathbf{A}$ and $\mathbf{B}$, move along an avenue while two other vehicles, $\mathbf{C}$ and D, are approaching from a cross street. At the intersection, a relay node $(\mathbf{R})$ supposedly within the range of all vehicles is installed. Vehicles A and $\mathbf{B}$ (similarly, $\mathbf{C}$ and $\mathbf{D}$ ) are close enough to have a direct link between them, but due obstacles (e.g., buildings) located at the corners, vehicles from one group can only exchange information with vehicles from the other group through the relay.

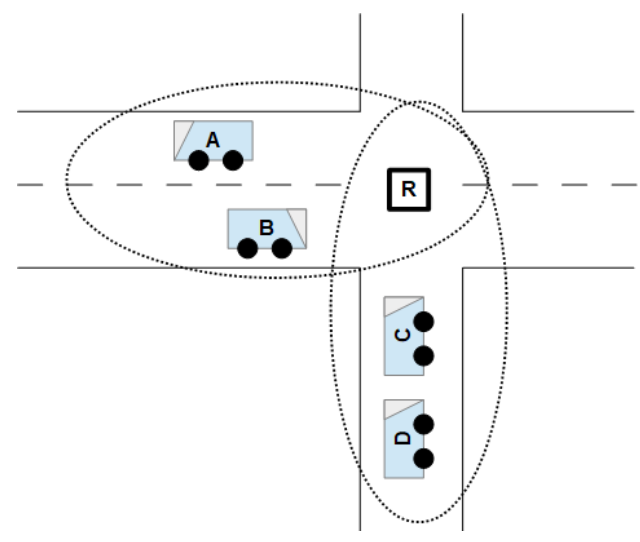

Fig. 1. Example of a mRN applied to a VANET scenario.

In the present work, a generalization of the system models introduced in [1], [2], [8], [9], called generalized multiway relay network $(\mathrm{g}-\mathrm{mRN})$, is proposed with the purpose of representing a broader selection of practical scenarios, as the one presented in Figure 1. Regarding the example, in g-mRN the four vehicles form a cluster, because they want to exchange their information. Vehicles that are close enough to have direct links are entitled to form a subcluster.
We introduce three new transmission strategies for $\mathrm{g}-\mathrm{mRN}$, all of them under the same framework of the MAC and BC phases. The transmission strategies differ only in the MAC phase. In the BC phase, the relay, after decoding each signal, combines the messages of different users in $\mathrm{GF}(2)$ using Network Coding (NC) [17] to transmit to all users.

The first transmission strategy is called massive-mRN, in which all users of the cluster transmit simultaneously in the first TS. Note that a user receives the signals from its neighbors in the same subcluster. If successfully recovered, these neighbors information becomes available to that user. This fact is not exploited in other works that also consider simultaneous transmission of several users, such as [5], [8], [9], highlighting the novelty of the proposed solution.

In the second transmission strategy, users transmit in pairs. It is named pairwise-mRN for that reason. An user from each different subcluster is selected for transmission in each TS. The relay receives a superposition of two signals (assuming two subclusters), and the users not transmitting in the current TS receive only the signal from the user selected for transmission in its corresponding subcluster. The transmission strategies in [3], [10]-[12] do not take advantage of this side information as we do here in our pairwise strategy.

Our third transmission strategy is an intermediate version, because half of the users in the cluster transmit simultaneously in two TS. At least one user from each subcluster is selected for transmission. This transmission strategy, called partial$\mathrm{mRN}$, also exploits side information in a way which is not considered in [4], [6], [13].

The contributions of this paper are:

- The introduction of a generalized multiway relay network, named g-mRN. It is a generalization of the system models presented in [1], [2], [8], [9]. Users who want to exchange information are arranged in a cluster. According to these users capability of direct communication, subclusters are formed. This novel characteristic of the proposed system is in contrast with the ones proposed in [8], [9], since in these works there exists a direct link between every pair of nodes in the system, regardless the cluster they belong.

- The introduction of three transmission strategies for g$\mathrm{mRN}$. Among the proposed transmission strategies, the performance of massive-mRN is the best when evaluated for the optimum message rate. However, for the case of a fixed message rate, the best transmission strategy depends on the particular rate and channel condition.

The rest of the paper is organized as follows. In Section II, g-mRN is detailed. The proposed transmission strategies are described in Section III. Next, in Section IV the concepts of effective rate and message rate, which are used to evaluate the performance of the transmission strategies, are defined. In Section V, the capacity region for each transmission strategy is presented. Then, in Section VI, numerical results considering the optimal message rate, as well as fixed message rates, are shown. Finally, Section VII brings the main conclusions and ideas for future works. 


\section{The Generalized Multiway Relay Network}

We consider a wireless network consisting of several users and a relay node. Users who want to exchange information among themselves are arranged in a cluster. In general, several clusters are possible, but it is assumed that their communications are mutually orthogonal (in time or frequency, for instance). So, we focus on a system with a single cluster. All nodes in the network communicate through full-duplex radios. This technology has become feasible as demonstrated for instance in [18], where self-interference cancellation of the order of $100 \mathrm{~dB}$ has been obtained experimentally.

A general system model for a multiway relay network is proposed in Figure 2, where a single cluster is shown. In this model, users which are in the range of each other (hence, can communicate directly) are organized in subclusters. The cases where a user could belong to different subclusters have to be resolved by the control center. So, herein we assume that each user belongs to a single subcluster.

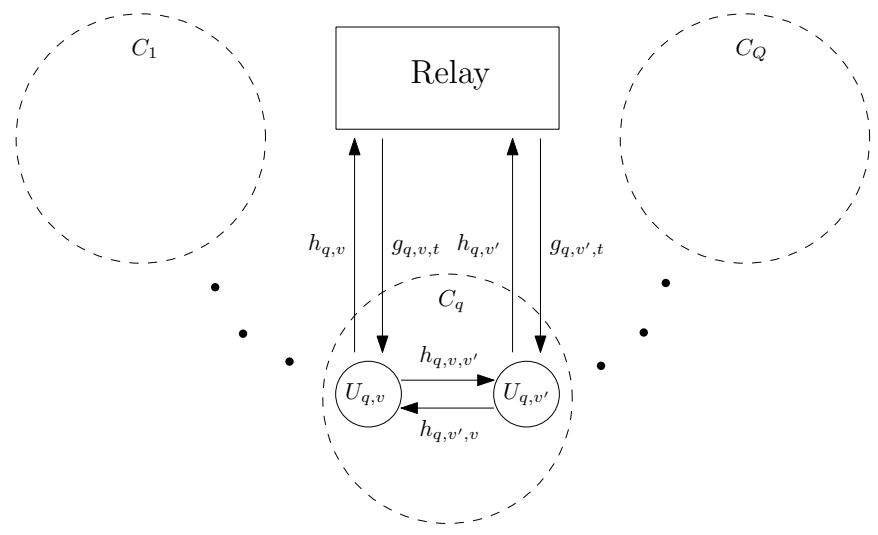

Fig. 2. A cluster in the generalized multiway relay network (g-mRN)

In Figure 2, $\mathrm{C}_{q}$ represents subcluster $q$, where $q \in \mathscr{Q}=$ $\{1, \ldots, Q\}$ and $Q$ is the number of subclusters. $\mathrm{U}_{q, v}$ denotes user $v$ of subcluster $q$, where $v \in \mathscr{V}=\left\{1, \ldots, V_{q}\right\}$ and $V_{q}$ is the number of users in subcluster $q$. The total number of users in the cluster is $M=\sum_{q=1}^{Q} V_{q}$.

The complex channel gain of the upload channel formed from user $\mathrm{U}_{q, v}$ to the relay is denoted by $h_{q, v}$. The complex channel gain of the channel formed from user $\mathrm{U}_{q, v}$ to user $\mathrm{U}_{q, v^{\prime}}$ is denoted by $h_{q, v, v^{\prime}}$. The complex channel gain of the download channel formed from the relay to user $\mathrm{U}_{q, v}$ in $\mathrm{TS}$ $t$ is denoted by $g_{q, v, t}$, where the range of $t$ depends on the transmission strategy employed. It should be noted that in all transmission strategies proposed in this work, all users transmit only once. However, depending on the strategy, the relay may need more than one TS to accomplish its goal. This explains the extra index in the download channel gain.

All channel gains are considered independent, complex Gaussian distributed with zero mean and unit variance. The gains remain fixed during a TS, and vary independently from one TS to another. In other words, the received signals are affected by quasi-static Rayleigh fading. They are also affected by additive white Gaussian noise (AWGN) with zero mean and variance $\sigma_{0}^{2}=N_{0} / 2$ per complex dimension.
In our analysis, we consider the outage model in which the individual channels as well as the multiple access and the broadcast channels are in outage whenever the transmission rate exceeds the channel capacity or the capacity region. We elaborate on that in a proper section later on.

\section{TRANSMission STRATEgIES}

The choice of the transmission strategy to be used in a $\mathrm{mRN}$ has a direct impact on its performance, its information transfer rate, and the complexity of its receivers. This is because each transmission strategy requires a minimum number of TS to complete the exchange of information among the users and also resorts to different numbers of simultaneous transmissions, which creates a trade-off between the effective rate and the detection complexity in the receivers.

For the proposed g-mRN, three transmission strategies are introduced. It should be mentioned that the number of TS indicated in each transmission strategy assumes that all transmissions are successful. However, the effective rate analysis to be derived in Section IV takes the possible transmissions failures into account.

\section{A. Pairwise-mRN}

In this transmission strategy, in the MAC phase users transmit in pairs, each user from a different subcluster. So, the number of TS is directly related to the number of users in the cluster. The relay receives the superposition of only two signals in each TS, and performs maximum likelihood detection [19], [20] to recover the information from both users. A user in the same subcluster of a transmitting user receives only its neighbor's signal. In the case where $M$ is odd, a single user may need to transmit all alone at the end of the MAC phase.

At the end of the MAC phase, the relay will have the information from all the $M$ users in the cluster while, individually, each user node will only have the information from the users in its subcluster. It is the relay's duty in the $\mathrm{BC}$ phase to let each user have the information from all the other users in the cluster. This is done with binary network coding [21].

Figure 3 illustrates the MAC phase of the pairwise-mRN transmission strategy with $Q=2$ subclusters and $V_{1}=V_{2}=3$ users in each subcluster. It takes 3 TS to be completed. In the first TS, user $\mathrm{U}_{1,1}$ (from subcluster $\mathrm{C}_{1}$ ) and user $\mathrm{U}_{2,1}$ (from subcluster $\mathrm{C}_{2}$ ) transmit simultaneously. In subcluster $\mathrm{C}_{1}$, users $\mathrm{U}_{1,2}$ and $\mathrm{U}_{1,3}$ receive the signal from $\mathrm{U}_{1,1}$ and try to recover its information, $x_{1,1}$. Similarly, in subcluster $\mathrm{C}_{2}$, users $\mathrm{U}_{2,2}$ and $\mathrm{U}_{2,3}$ try to recover the information, $x_{2,1}$. The relay receives the superposition of the signals coming from $\mathrm{U}_{1,1}$ and $\mathrm{U}_{2,1}$, and tries to recover the two information, $x_{1,1}$ and $x_{2,1}$, which can be done with maximum likelihood (ML) detection [19], [20]. The same process is repeated for the pairs of users $\left(\mathrm{U}_{1,2}, \mathrm{U}_{2,2}\right)$ and $\left(\mathrm{U}_{1,3}, \mathrm{U}_{2,3}\right)$ in the two subsequent TS, as shown in Figure 3. Note that, at the end of the MAC phase, each user will have the information of its two neighbors in the subcluster, while the relay will have the information of all users in the cluster. 


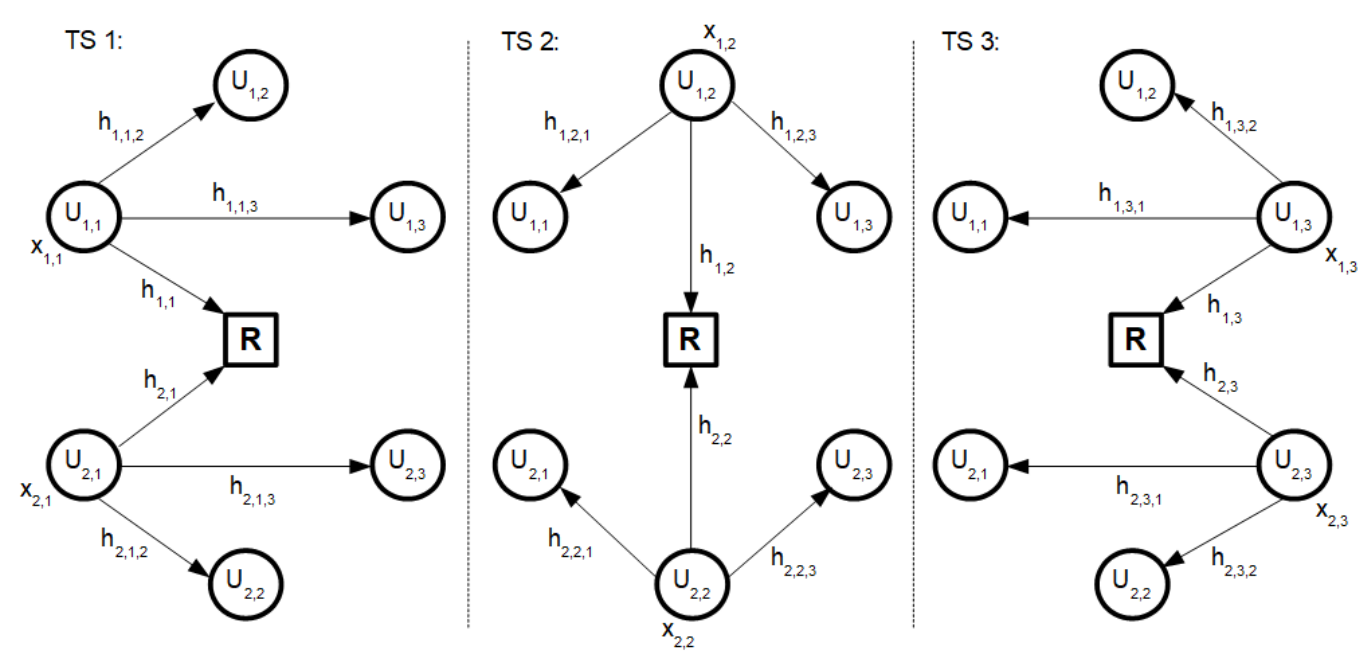

Fig. 3. Example of the MAC phase of the transmission strategy pairwise-mRN with three users in each one of the two subclusters.

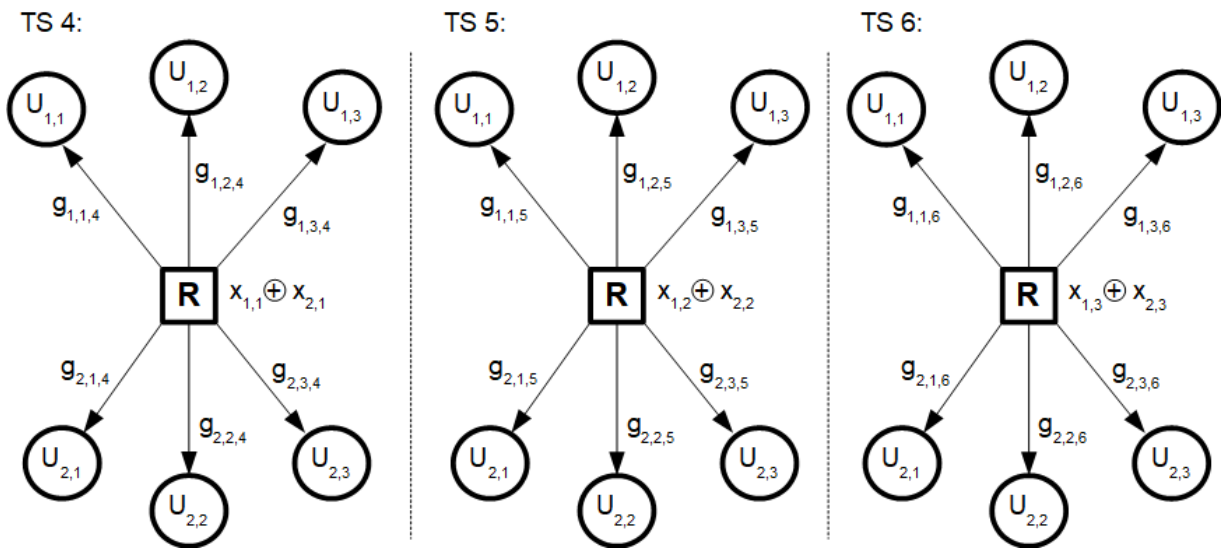

Fig. 4. Example of the $\mathrm{BC}$ phase with six users in the cluster with two subclusters.

In the $\mathrm{BC}$ phase, the relay produces three different binary linear combinations of the six messages and then broadcasts them in three TS to all users, as shown in Figure 4. The linear combinations are: $x_{1,1} \oplus x_{2,1}, x_{1,2} \oplus x_{2,2}$, and $x_{1,3} \oplus x_{2,3}$. Each user forms a system with these three linear equations. Since the user knows three out of the six unknowns, it is able to solve the system [21].

It should be remarked that, in all transmission strategies considered in this work, at the end of the MAC phase each user will have its own information as well as the ones from its neighbors in the subcluster. Therefore, the $\mathrm{BC}$ phases in all transmission strategies are exactly the same.

Note that, in general, with $M=\sum_{q=1}^{Q} V_{q}$ users in the cluster, the relay must transmit $N_{\mathrm{TS}}^{\mathrm{BC}}=M-\min _{q \in \mathcal{Q}}\left\{V_{q}\right\}$ independent linear combinations of the $M$ messages in $N_{\mathrm{TS}}^{\mathrm{BC}}$ time slots. In this way, user $\mathrm{U}_{q, v}$ will be able to form a system with $N_{\mathrm{TS}}^{\mathrm{BC}}$ equations and $M$ unknowns. But, since this user has knowledge of $V_{q}$ unknowns, it is able to solve the system for all the $M$ messages.

The pairwise-mRN strategy requires $\frac{M}{2}$ TS for the MAC phase (assuming $M$ even) and $N_{\mathrm{TS}}^{\mathrm{BC}} \mathrm{TS}$ for the $\mathrm{BC}$ phase, resulting in $\frac{3 M}{2}-\min _{q \in \mathscr{Q}}\left\{V_{q}\right\}$ TS in total for the users of a cluster to exchange their information among themselves.

\section{B. Massive- $m R N$}

In this transmission strategy, the MAC phase consists of all users in the cluster transmitting simultaneously in a single TS. Therefore, the relay receives a superposition of $M$ signals and each user in subcluster $\mathrm{C}_{q}$ receives a superposition of $V_{q}-1$ signals. Similar to the pairwise-mRN strategy, at the end of the MAC phase each user will have the information of all users in its subcluster, while the relay will have the information of all users in the cluster. Herein, we assume multiuser ML detection in all receivers. An illustration of the MAC phase of massive$\mathrm{mRN}$, with two subclusters and three users per subcluster, is shown in Figure 5.

As already explained, the $\mathrm{BC}$ phase of massive- $\mathrm{mRN}$ is again the one in Figure 4. It should be noted that with the same scenario of two subclusters and three users per subcluster, massive-mRN requires only four TS, two less than does pairwise-mRN. On the other hand, the relay has to detect the superposition of six users, as opposed to two of pairwise$\mathrm{mRN}$. Therefore, a comprehensive analysis is in order. 


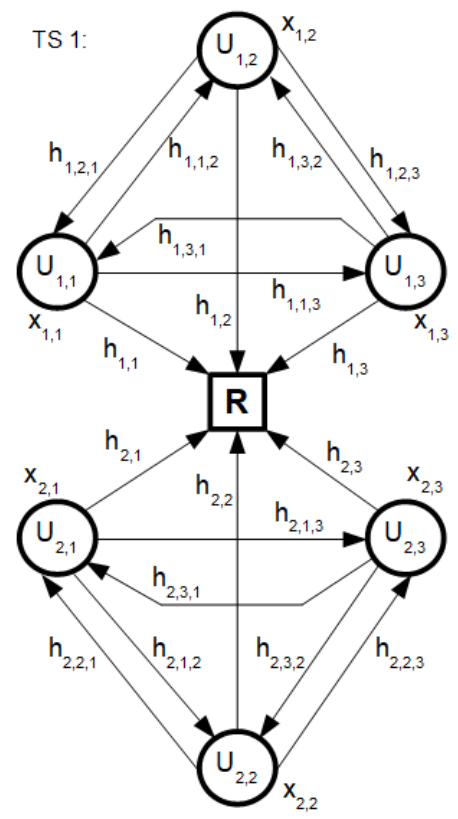

Fig. 5. Example of the MAC phase of the transmission strategy massive-mRN with 6 users in the network.

In general, massive-mRN requires $1+N_{\mathrm{TS}}^{\mathrm{BC}} \mathrm{TS}$ in total for all the $M$ users in the cluster to exchange information among themselves.

\section{Partial-mRN}

As a way of reducing the number of superimposed signals and hence alleviate the decoding complexity, this transmission strategy allows the participation of only a small number of users (greater than 2 but smaller than $M$ ) in simultaneous transmissions. Several configurations are possible and for each one a particular analysis is required.

Herein, we adopt the restriction in partial-mRN that its MAC phase has to have 2 TS. In this way, with $M$ users distributed among $Q$ subclusters, about half of the users in each subcluster transmit in the first TS, and the other users transmit in the second TS. We illustrate the idea with an example.

For the same scenario with six users equally distributed among two subclusters, the MAC phase of partial-mRN is shown in Figure 6. In the first TS, users $\mathrm{U}_{1,1}, \mathrm{U}_{1,3}$, and $\mathrm{U}_{2,2}$ are selected for transmitting simultaneously. Note that there are two users in subcluster $\mathrm{C}_{1}$ and one user from subcluster $\mathrm{C}_{2}$. In the second TS, the remaining users transmit. Clearly, by performing either single user or multiuser ML detection, at the end of the MAC phase each user will have the information of all users in its subcluster, while the relay will again have information from all users in the cluster. The BC phase in Figure 4 may then begin.

It should be noted that partial-mRN uses two TS in its MAC phase and the maximum number of superimposed signals received by the relay is three (in this example). Therefore, this transmission strategy lies between the two ones already introduced. These characteristics will be taken into account in the analysis of the next section.

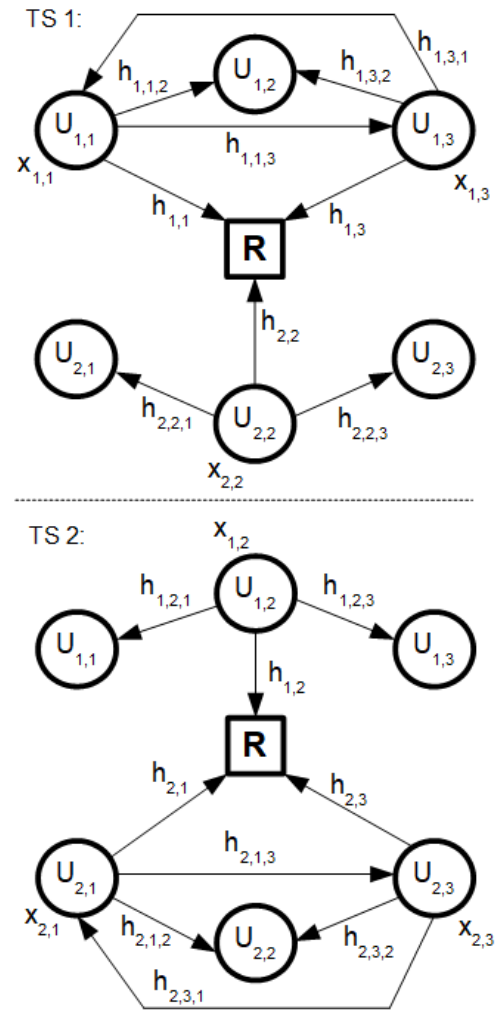

Fig. 6. Example of the MAC phase of the transmission strategy partial-mRN with six users in the network.

\section{Comparing the Transmission Strategies}

Two parameters are important in the comparison of the transmission strategies, namely, the number of TS required and the number of signals simultaneously transmitted by users. The former has a direct impact on the information transfer rate, while the latter directly affects the performance and the complexity of receivers.

For a g-mRN consisting of one cluster, two subclusters, and $M$ users equally distributed in the two subclusters, we list in Tables I and II these important parameters for the three transmission strategies introduced in this work. More specifically, Table I presents the minimum number of TS required by the strategy, i.e., assuming no transmissions failures. Table II presents the maximum number of superimposed signals received by a user or the relay.

TABLE I

MINIMUM NUMBER OF TS

\begin{tabular}{|c|c|c|c|}
\hline$M$ & pairwise-mRN & massive-mRN & partial-mRN \\
\hline 4 & 4 & 3 & 4 \\
\hline 6 & 6 & 4 & 5 \\
\hline 8 & 8 & 5 & 6 \\
\hline 10 & 10 & 6 & 7 \\
\hline 12 & 12 & 7 & 8 \\
\hline
\end{tabular}

It is important to note that pairwise-mRN requires the largest number of TS. On the other hand, the users in this transmission strategy need not perform multiuser detection. The maximum number of superimposed signals is the largest 
TABLE II

MAXIMUM NUMBER OF SUPERIMPOSED SIGNALS ARRIVING IN A USER (U) AND IN THE RELAY (R)

\begin{tabular}{|c|cc|cc|cc|}
\hline$M$ & \multicolumn{2}{|l|}{ pairwise- } & \multicolumn{2}{|l|}{ massive- } & \multicolumn{2}{|l|}{ partial- } \\
mRN & mRN & \multicolumn{2}{|c|}{ mRN } \\
\hline & U & $\mathbf{R}$ & U & R & U & R \\
\hline 4 & 1 & 2 & 1 & 4 & 1 & 2 \\
\hline 6 & 1 & 2 & 2 & 6 & 2 & 3 \\
\hline 8 & 1 & 2 & 3 & 8 & 2 & 4 \\
\hline 10 & 1 & 2 & 4 & 10 & 3 & 5 \\
\hline 12 & 1 & 2 & 5 & 12 & 3 & 6 \\
\hline
\end{tabular}

in massive-mRN, demanding a high effort by the relay and the users. In general, under this circumstance, the decoding performance tends to deteriorate. However, since the MAC phase in this transmission strategy consists of a single TS, the exchange of information among users is completed in the least time. As already discussed, partial-mRN situates itself between the other two strategies. These trade-offs are in the core of the analysis presented next.

\section{Maximum Effective Rate}

The transmission strategies are now evaluated in terms of effective rate, an efficiency parameter presented in [22] for two-way relay channel (TWRC). This concept is now generalized for a network with multiples users, in accordance with the system model introduced in Figure 2.

\section{A. Message Rate}

Consider that each user has the same number of information bits, $B$, to transmit. Each block of bits is divided into $K$ packets of information, each one containing $k=\frac{B}{K}$ bits. Each information packet is coded, resulting in a packet of $n$ complex symbols. Thus, the message rate, $r$, is defined as [22]

$$
r=\frac{k}{n} \quad\left[\frac{\text { information bits }}{\text { complex symbols }}\right] \text {. }
$$

\section{B. Effective Rate}

The effective rate is the data rate from a user point of view taking into account possible transmission losses. It is defined as [22]

$$
R=\frac{B}{n E[N]} \quad\left[\frac{\text { information bits }}{\text { complex symbols }}\right],
$$

where $E[\cdot]$ denotes expectation and $N$ is the total number of transmitted packets until the $B$ information bits are successfully decoded by all intended receivers.

Note that $N$ depends on the channel state quality. Hence, it is a random variable. Similarly to [22], if $p$ is the success probability of a transmitted packet, then we have $E[N]=\frac{K}{p}$. Considering that the coded packets are sufficiently long so that channel coding takes effect, the success probability $p$ for a point-to-point channel is given as [22]

$$
p=p(r)=P(r<C),
$$

where $C$ is the instantaneous channel capacity [20]. For other channels, such as the multiple access channel, the success event given by the inequality $r<C$ may be substituted by a set of inequalities (called capacity region). In an even broader sense, the success event for the MAC and the BC phases of a transmission strategy is described as a set of constraints, which will be presented in Section V.

The effective rate can be rewritten as [22]

$$
R(r)=p(r) \cdot r,
$$

which is a function of the message rate and its corresponding success probability. Note that the success probability is a decreasing function of the message rate. Therefore, there is an optimal value of the message rate that maximizes the effective rate, which is called maximum effective rate and is defined in [22] as

$$
R^{*}=\max _{r>0}\{p(r) \cdot r\} .
$$

The value of the message rate that maximizes the effective rate is called optimal message rate, and is defined in [22] as

$$
r^{*}=\arg \max _{r>0}\{p(r) \cdot r\} .
$$

\section{Effective Rate of the Proposed Transmission Strategies}

For the analysis of the effective rate of the transmission strategies proposed for the g-mRN (Figure 2), we must separate the phases MAC and $\mathrm{BC}$ for two reasons: 1) In case of failure during the $\mathrm{BC}$ phase, the repetition of the MAC phase is not necessary; 2) The rate optimizations in (5) and (6) are independent in the two phases, allowing the adoption of different optimal message rates in each phase.

It is also possible to optimize the message rates on an individual basis, i.e., one optimal message rate for each user. We present our formulation of the effective rate analysis in that level of generality. However, in the numerical results of Section VI, we assume for simplicity that all users and the relay adopt the same message rate. In spite of this restriction, the numerical results are rich enough to provide a good understanding of the proposal.

A multiple access channel in the MAC phase for say $U$ users transmitting simultaneously is herein referred to as $\operatorname{MAC}(U)$. So, let $R_{\mathrm{MAC}(U)}^{*}$ denote the maximum effective rate of $\operatorname{MAC}(U)$. The MAC phase of a transmission strategy can consist of one or several MAC $(U)$ 's. Also, let $R_{\mathrm{BC}}^{*}$ denote the maximum effective rate of the $\mathrm{BC}$ phase. Then the maximum effective rate (see [22] for details) for pairwise- $\mathrm{mRN}$ is

$$
R_{\text {pairwise }}^{*}=M \cdot \frac{1}{\frac{M}{2 \cdot R_{\mathrm{MAC}(2)}^{*}}+\frac{M}{2 \cdot R_{\mathrm{BC}}^{*}}},
$$

for massive- $\mathrm{mRN}$ is

$$
R_{\text {massive }}^{*}=M \cdot \frac{1}{\frac{1}{R_{\mathrm{MAC}(M)}^{*}}+\frac{M}{2 \cdot R_{\mathrm{BC}}^{*}}},
$$

and for partial-mRN is

$$
R_{\text {partial }}^{*}=M \cdot \frac{1}{\frac{2}{R_{\mathrm{MAC}(M / 2)}^{*}}+\frac{M}{2 \cdot R_{\mathrm{BC}}^{*}}} .
$$


Equations (7), (8), and (9) put in evidence the fact that the MAC phase controls the maximum effective rate, since the BC phase is the same for all transmission strategies.

\section{CAPACITY REgion}

The instantaneous capacity of a point-to-point fading channel with a given average signal-to-noise ratio, SNR, is given by [19], [20]

$$
C(x) \triangleq \log _{2}(1+x \cdot \mathrm{SNR}) \quad[\text { bits/complex symbols }],
$$

where $x$ is the square modulus of the instantaneous channel gain.

The capacity region of $\operatorname{MAC}(U)$ is described by $\left(2^{U}-1\right)$ constraints, one for each non-empty subset of the set of indices of users [20], $\mathscr{U}=\{1, \ldots, U\}$. For the BC phase, the capacity region is the same regardless of the transmission strategy chosen, and is basically expressed by the instantaneous capacity of all point-to-point channels formed from the relay to all users. Recall that, for the downlink, the channel gains are denoted by $g_{q, v, t}$, where $q, v$, and $t$ refer to subcluster, user, and TS, respectively [22].

For simplicity of presentation, we assume the same number of users in each subcluster. The set of indices of subclusters and the set of indices of users in a subcluster are $\mathscr{Q}=\{1, \ldots, Q\}$ and $\mathscr{V}=\{1, \ldots, V\}$, respectively, where $V=M / Q$.

Consider a partition of the set $\{(q, v): q \in \mathscr{Q}, v \in \mathscr{V}\}$ into $\frac{M}{2}$ subsets of two elements each, defined by:

$$
\mathscr{P}_{i}=\left\{\left(q_{1}^{(i)}, v_{1}^{(i)}\right),\left(q_{2}^{(i)}, v_{2}^{(i)}\right)\right\}
$$

with the restriction that $q_{1}^{(i)} \neq q_{2}^{(i)}$, for $i=1, \ldots, \frac{M}{2}$.

The capacity region of the pairwise-mRN MAC phase is given by

$$
\begin{aligned}
& \left\{\left(r_{q, v}\right), \forall q \in \mathscr{Q}, \forall v \in \mathscr{V}:\right. \\
& \left.r_{q, v}<C\left(\left|h_{q, v, v^{\prime}}\right|^{2}\right), \forall v^{\prime} \in \mathscr{V}, v^{\prime} \neq v\right\} \text {, } \\
& \left\{r_{q_{1}^{(i)}, v_{1}^{(i)}}, r_{q_{2}^{(i)}, v_{2}^{(i)}}, \forall i \in\{1, \ldots, M / 2\}:\right. \\
& r_{q_{1}^{(i)}, v_{1}^{(i)}}<C\left(\left|h_{q_{1}^{(i)}, v_{1}^{(i)}}\right|^{2}\right) \\
& r_{q_{2}^{(i)}, v_{2}^{(i)}}<C\left(\left|h_{q_{2}^{(i)}, v_{2}^{(i)}}\right|^{2}\right) \\
& \left.r_{q_{1}^{(i)}, v_{1}^{(i)}}+r_{q_{2}^{(i)}, v_{2}^{(i)}}<C\left(\sum_{j=1}^{2}\left|h_{q_{j}^{(i)}, v_{j}^{(i)}}\right|^{2}\right)\right\}
\end{aligned}
$$

Note that sub-equations (12a)-(12b) consider the transmission from a user to its neighbors in the subcluster, while subequations (12c)-(12f) consider the transmission from the users to the relay.

The capacity region of the massive-mRN MAC phase is

$$
\begin{gathered}
\left\{\left(r_{q, v}\right), \forall q \in \mathscr{Q}, \forall v \in \mathscr{V}:\right. \\
\sum_{s \in \mathscr{S}} \sum_{u \in \mathscr{U}} r_{s, u}<C\left(\sum_{s \in \mathscr{S}} \sum_{u \in \mathscr{U}}\left|h_{s, u}\right|^{2}\right), \\
\forall \mathscr{S} \subseteq \mathscr{Q}, \forall \mathscr{U} \subseteq \mathscr{V} ;
\end{gathered}
$$

$$
\begin{aligned}
& \sum_{u \in \mathscr{U}} r_{q, u}<C\left(\sum_{u \in \mathscr{U}}\left|h_{q, u, v^{\prime}}\right|^{2}\right), \\
&\left.\forall \mathscr{U} \subseteq \mathscr{V} \backslash\left\{v^{\prime}\right\}, v^{\prime} \in \mathscr{V}\right\} .
\end{aligned}
$$

In this case, sub-equation (13b) considers the simultaneous transmission of all users in the network to the relay, while sub-equation $(13 \mathrm{c})$ refers to the simultaneous transmissions and receptions which occur within the subcluster.

A general expression for the capacity region of partial$\mathrm{mRN}$ is difficult to obtain due to the several possibilities of configurations. Therefore, we present next the result for the case where there are only two subclusters, and the MAC phase consists of two TS. In the first TS, about half of the users in each subcluster transmit simultaneously. In the second TS, the other half of the users in each subcluster transmit. This configuration for two subclusters and three users in each subcluster was shown in the example considered in Figure 6. Despite this limitation, this result sheds some light on the analyses of other possible configurations.

The capacity region of the partial-mRN MAC phase, considering our assumption of two subclusters and two TS in this phase, is

$$
\begin{gathered}
\left\{\left(r_{q, v}\right), \forall q \in\{1,2\}, \forall v \in \mathscr{V}:\right. \\
\sum_{u \in \mathscr{U}} r_{1, u}+\sum_{\substack{u^{\prime} \in \mathscr{U} \\
\forall}} r_{2, u^{\prime}}<C\left(\sum_{u \in \mathscr{U}}\left|h_{1, u}\right|^{2}+\sum_{u^{\prime} \in \mathscr{U}}\left|h_{2, u^{\prime}}\right|^{2}\right), \\
\forall \mathscr{U} \subseteq\{1, \ldots,\lceil V / 2\rceil\}, \forall \mathscr{U}^{\prime} \subseteq\{1, \ldots,\lfloor V / 2\rfloor\} ;
\end{gathered}
$$

$$
\begin{array}{r}
\sum_{u \in \mathscr{U}} r_{1, u}+\sum_{u^{\prime} \in \mathscr{U}^{\prime}} r_{2, u^{\prime}}<C\left(\sum_{u \in \mathscr{U}}\left|h_{1, u}\right|^{2}+\sum_{u^{\prime} \in \mathscr{U}^{\prime}}\left|h_{2, u^{\prime}}\right|^{2}\right), \\
\forall \mathscr{U} \subseteq\{\lceil V / 2\rceil+1, \ldots, V\}, \\
\forall \mathscr{U}^{\prime} \subseteq\{\lfloor V / 2\rfloor+1, \ldots, V\} ;
\end{array}
$$

$$
\begin{array}{r}
\sum_{u \in \mathscr{U}} r_{q, u}<C\left(\sum_{u \in \mathscr{U}}\left|h_{q, u, v^{\prime}}\right|^{2}\right), \\
\forall \mathscr{U} \subseteq\{1, \ldots,\lceil V / 2\rceil\}, v^{\prime} \in\{\lceil V / 2\rceil+1, \ldots, V\} ; \\
\sum_{u^{\prime} \in \mathscr{U} \prime^{\prime}} r_{q, u^{\prime}}<C\left(\sum_{u^{\prime} \in \mathscr{U}}\left|h_{q, u^{\prime}, v^{\prime}}\right|^{2}\right), \\
\left.\forall \mathscr{U}^{\prime} \subseteq\{1, \ldots,\lfloor V / 2\rfloor\}, v^{\prime} \in\{\lfloor V / 2\rfloor+1, \ldots, V\}\right\} .
\end{array}
$$

Sub-equation (14b) is related to the first selected users from each subcluster transmitting to the relay (first TS). Note that if there is an odd number of users transmitting simultaneously, as in the example presented in the Figure 6, then subcluster $\mathrm{C}_{1}$ has one more user transmitting during the first TS. In the second TS, it is subcluster $\mathrm{C}_{2}$ that has one more user transmitting simultaneously. Thus, sub-equation (14c) considers the users transmitting to relay during the second TS. The next two sub-equations, (14d) and (14e), refer to the users transmitting simultaneously to other users in the same subcluster, during the first and the second TS, respectively.

Finally, the capacity of the BC phase is

$$
\begin{array}{r}
\left\{\left(r_{q, v}\right), \forall q \in \mathscr{Q}, \forall v \in \mathscr{V}:\right. \\
\left.\left(r_{q, v}\right)<C\left(\left|g_{q, v, t+N_{\text {TS }}^{\text {MAC }}}\right|^{2}\right), \forall t \in\left\{1, \ldots, N_{\text {TS }}^{\mathrm{BC}}\right\}\right\},
\end{array}
$$


where $N_{\mathrm{TS}}^{\mathrm{MAC}}$ is the number of TS in the corresponding MAC phase.

Upon meeting all the constraints described above, the communication is said to be successful [19], [20].

\section{NUMERICAL RESULTS}

A theoretical analysis, involving the expectation of all inequality constraints with regard to the channel statistics, is extremely difficult to obtain. Therefore, we resort to Monte Carlo simulation in order to obtain the maximum effective rate and the optimal message rate for the transmission strategies. For simplicity, we assume $r_{q, v}=r$, for all $q \in \mathscr{Q}$ and $v \in \mathscr{V}$.

The process to compute the maximum effective rate is the following. From several independent channel realizations, and for a given rate $r$, we can obtain an estimate of the success probability through the fraction of channel realizations satisfying the constraints (capacity regions) presented in the previous section. Recall that the MAC and the BC phases are independent; different outcomes are expected to occur. By varying the message rate, $r$, and repeating the process above to obtain the associated success probabilities, we can obtain the maximum effective rate and the optimal message rate through (5) and (6), respectively, for both the MAC and the BC phases. Finally, the maximum effective rate is obtained from equations (7), (8) or (9), depending on the transmission strategy.

We consider $M=4,6$, and 8 users in the network. The SNR varies from 0 to $50 \mathrm{~dB}$, and for each one of these values we consider 10,000 channel realizations for obtaining the success probability.

\section{A. Maximum Effective Rate Results}

The results for the maximum effective rate of each proposed transmission strategy versus SNR for the various values of $M$ are presented in Figure 7. Clearly, massive-mRN is the transmission strategy with the best maximum effective rate. This is due to the fact that it requires fewer TS to complete the information exchange than its competitors.

The results for the optimal message rates of the MAC and the $\mathrm{BC}$ phases are presented in Figure 8. It can be seen that massive-mRN operates with the lowest optimal message rate. Although this implies less information per transmitted packet, the use of less TS for completing the communication process compensates this loss.

\section{B. Effective Rate for a fixed Message Rate}

If the instantaneous SNR is not available to the transmitters, they are not able to use the optimal message rate. In this case, a fixed message rate has to be adopted, and the maximum effective rate of the previous subsection represents an upper bound for the effective rate of the system.

In this subsection, we evaluate the effective rate under the following fixed message rates: $r=1,2,3$, and 4 , for both the MAC and the BC phases. The number of users is fixed to $M=6$. The effective rate of each transmission strategy and each fixed message rate is presented in Figure 9. The maximum effective rate as a benchmark is also plotted.
As can be seen from the figure, the best transmission strategy is a function of both the message rate and the SNR. The typical behavior is that the effective rate is zero for low values of SNR up to a certain threshold. Then the effective rate begins to increase as the SNR gets higher, up to a point beyond which it saturates.

Massive-mRN is the best strategy (but very similar to partial-mRN) for $r \leq 1$. Partial-mRN has the highest effective rate for $2 \leq r \leq 3$, for practically all SNRs, except for very high SNR $(>48 \mathrm{~dB})$, in which case massive-mRN is better. For higher message rates $(r \geq 4)$, all three strategies halt for SNR smaller than $30 \mathrm{~dB}$, which means that in a practical system message rates should never be that high.

In the absence of knowledge of the instantaneous SNR, but assuming that the SNR value in a typical wireless communication system ranges from 15 to $35 \mathrm{~dB}$, a reasonable choice is a message rate between 2 and 3 used in conjunction with partial-mRN.

It should be noted that some effective rates in Figure 9 touch or nearly touch the maximum effective rate for some specific values of SNR. H when the fixed message rate, $r$, is very similar to the optimal message rate, $r^{*}$, of the transmission strategy.

Finally, we have obtained results (not shown) for $M$ equal to 4 and 8 . The same relative behavior of the effective rate for the three strategies has been observed. We only want to remark that the effective rates approach the upper bound for fewer users and move away from it as the number of users increases.

\section{CONCLUSION}

In this paper, the g-mRN system model, which covers a wide range of practical topologies, has been introduced. Three new transmission strategies for this network, namely, pairwise$\mathrm{mRN}$, massive-mRN, and partial-mRN, have been proposed. In these strategies, users transmit to the relay and to some of the others users, those in the same subcluster. This helps decode the information during the $\mathrm{BC}$ phase.

The effective rate has been optimized and, for a known instantaneous SNR, the maximum effective rate has been obtained. The transmission strategy massive-mRN obtained the best maximum effective rate for the SNR range studied. However, when the SNR is not know, the best transmission strategy depends on both the message rate adopted in the transmitter and the SNR value. The strategy partial-mRN with a message rate between 2 and 3 seems to be a good choice in a practical scenario when the instantaneous SNR value is not known.

The transmission strategies presented are suitable for scenarios such as VANETs and WSNs, in which the presence of clusters and subclusters is natural. Thus, the g-mRN and the associated transmission strategies can be applied to several practical applications. As future work, an energy consumption analysis of $\mathrm{g}-\mathrm{mRN}$, for a WSN scenario, can be considered. The idea is to find the transmission strategy with highest effective rate under the restriction of maintaining a reasonably long battery lifetime. As another proposal for future work, 


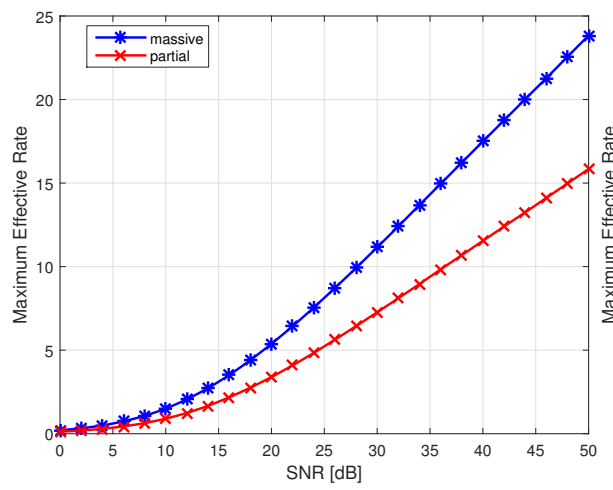

(a) $M=4$

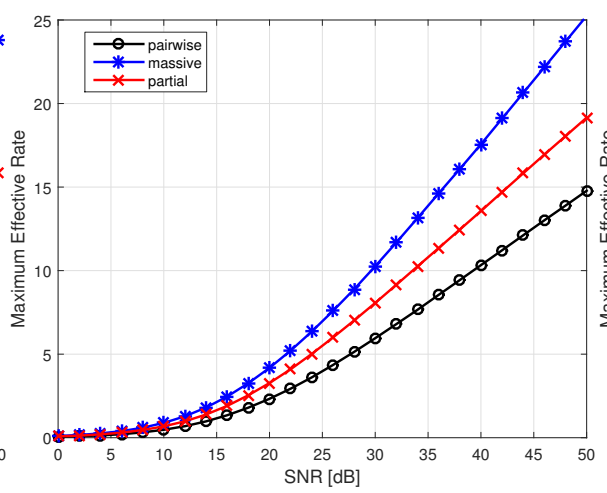

(b) $M=6$

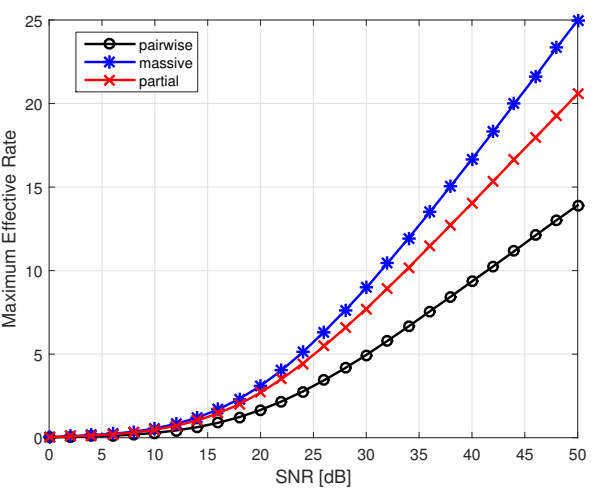

(c) $M=8$

Fig. 7. Maximum effective rates of the three transmission strategies, for different number of users.

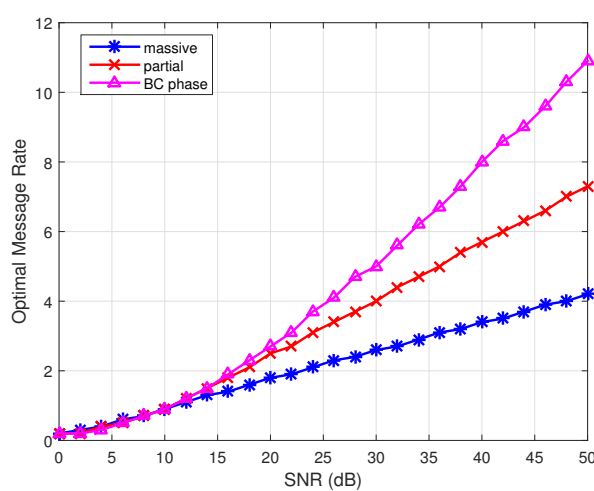

(a) $M=4$

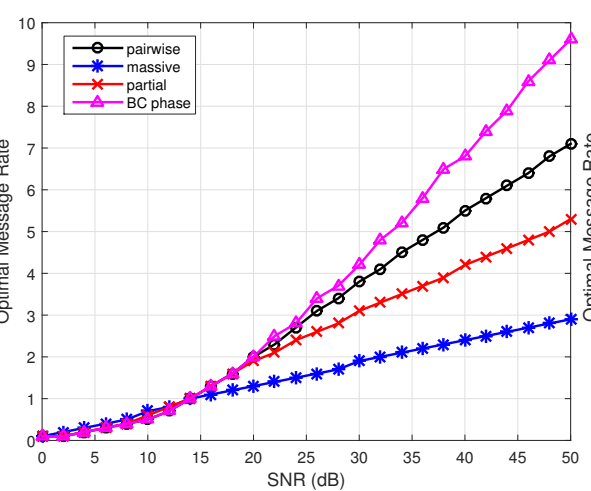

(b) $M=6$

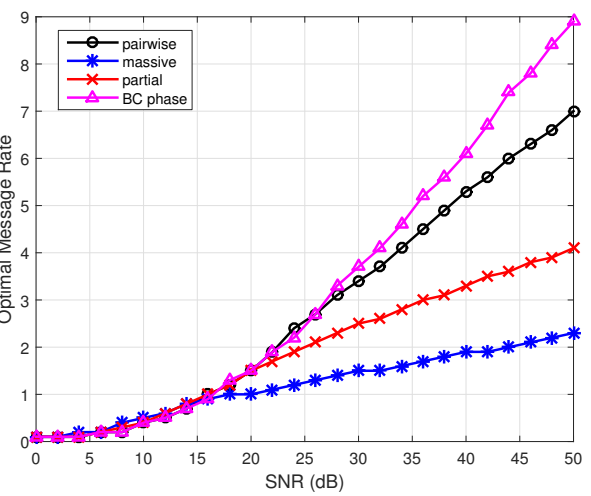

(c) $M=8$

Fig. 8. Optimal message rate (the same for all transmission strategies) of the $\mathrm{BC}$ phase and optimal message rates (a different one for each of the three transmission strategies) of the MAC phase, for different numbers of users.

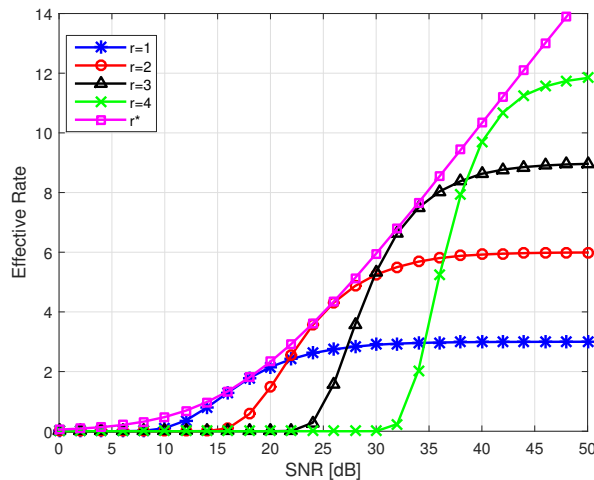

(a) pairwise-mRN

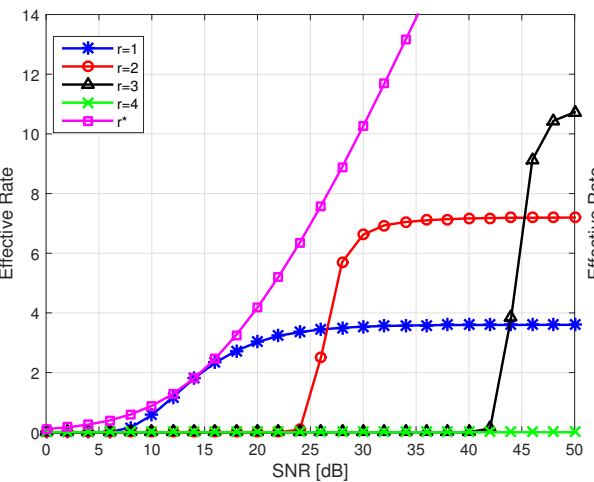

(b) massive-mRN

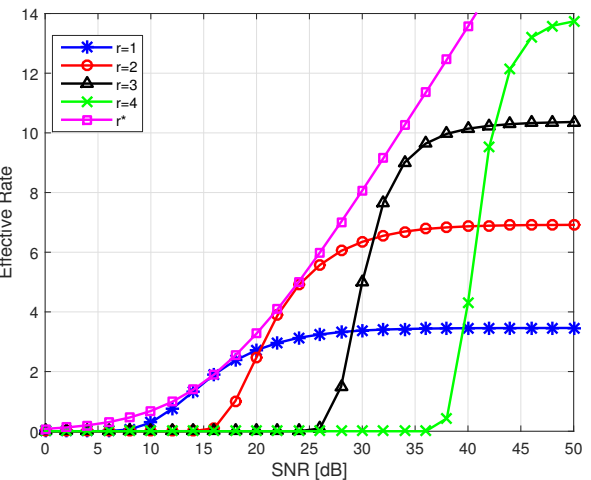

(c) partial-mRN

Fig. 9. Effective rates of the three transmission strategies for $M=6$ users, considering different message rates.

multiple antennas at the transmitters and/or receivers can be considered. This would be in accordance with the VANETs standards [14], [23], [24].

\section{REFERENCES}

[1] D. Gündüz, A. Yener, A. Goldsmith, and H. V. Poor, "The multi-way relay channel," in 2009 IEEE International Symposium on Information Theory, June 2009, pp. 339-343, doi: 10.1109/ISIT.2009.5205634.

[2] _ , "The multiway relay channel," IEEE Transactions on Information Theory, vol. 59, no. 1, pp. 51-63, Jan 2013, doi: 10.1109/TIT.2012. 2219156.
[3] S. N. Islam, S. Durrani, and P. Sadeghi, "A novel user pairing scheme for functional decode-and-forward multi-way relay network," Physical Communication, vol. 17, pp. 128 - 148, 2015. [Online]. Available: www.sciencedirect.com/science/article/pii/S1874490715000440. doi:http://dx.doi.org/10.1016/j.phycom.2015.08.009

[4] K. Anwar, "High-dense multiway relay networks exploiting direct links as side information," in 2016 IEEE International Conference on Communications (ICC), May 2016, pp. 1-6, doi: 10.1109/ICC.2016.7511178.

[5] L. Ong, S. J. Johnson, and C. M. Kellett, "An optimal coding strategy for the binary multi-way relay channel," IEEE Communications Letters, vol. 14, no. 4, pp. 330-332, April 2010, doi: 10.1109/LCOMM.2010. 04.092427 . 
[6] A. A. Purwita and K. Anwar, "Vehicular massive multiway relay networks applying graph-based random access," in 2015 IEEE Vehicular Networking Conference (VNC), Dec 2015, pp. 227-234, doi: 10.1109/VNC.2015.7385581.

[7] L. Ong, S. J. Johnson, and C. M. Kellett, "Optimal coding functions for pairwise message sharing on finite-field multi-way relay channels," in 2014 IEEE International Conference on Communications (ICC), June 2014, pp. 1866-1871, doi: 10.1109/ICC.2014.6883595.

[8] Y. Su, Y. Li, G. Song, and L. Liu, "Achievable rate regions of multi-way relay channel with direct links," IET Communications, vol. 9, no. 7, pp. 947-959, 2015, doi: 10.1049/iet-com.2014.0622.

[9] A. Savard and C. Weidmann, "On the gaussian multiway relay channel with intra-cluster links," EURASIP Journal on Wireless Communications and Networking, vol. 2016, no. 1, p. 267, 2016. [Online]. Available: doi:http://dx.doi.org/10.1186/s13638-016-0763-5

[10] R. R. Borujeny, M. Noori, and M. Ardakani, "On the achievable rates of pairwise multiway relay channels," in 2014 IEEE International Symposium on Information Theory, June 2014, pp. 2719-2723, doi: 10.1109/ISIT.2014.6875328.

[11] _ "Maximizing data rate for multiway relay channels with pairwise transmission strategy," IEEE Transactions on Wireless Communications, vol. 16, no. 3, pp. 1609-1618, March 2017, doi: 10.1109/TWC.2017. 2649521.

[12] S. N. Islam, "Error performance analysis of a clustered multiway relay network," IEEE Transactions on Vehicular Technology, vol. 65, no. 11, pp. 8952-8963, Nov 2016, doi: 10.1109/TVT.2016.2519507.

[13] M. N. Hasan and K. Anwar, "Massive uncoordinated multiway relay networks with simultaneous detections," in 2015 IEEE International Conference on Communication Workshop (ICCW), June 2015, pp. 21752180, doi: 10.1109/ICCW.2015.7247504.

[14] H. Hartenstein and K. P. Laberteaux, VANET: Vehicular Applications and Inter-Networking Technologies. John Wiley \& Sons, 2010, doi: 10.1002/9780470740637.

[15] B. B. Marc Emmelmann and C. C. Kellum, Vehicular Networking: Automotive Applications and Beyond. John Wiley \& Sons, 2010, doi: $10.1002 / 9780470661314$

[16] H. T. Cheng, H. Shan, and W. Zhuang, "Infotainment and road safety service support in vehicular networking: From a communication perspective," Mechanical Systems and Signal Processing, vol. 25, no. 6 , pp. 2020 - 2038, 2011, interdisciplinary Aspects of Vehicle Dynamics. [Online]. Available: doi:http://dx.doi.org/10.1016/j.ymssp.2010.11.009.

[17] R. W. Yeung, S.-y. Li, and N. Cai, Network Coding Theory (Foundations and Trends $(R)$ in Communications and Information Theory). Hanover, MA, USA: Now Publishers Inc., 2006, doi: http://dx.doi.org/10.1561/ 0100000007

[18] D. Bharadia, E. McMilin, and S. Katti, "Full duplex radios," in Proc od the 2013 ACM SIGCOMM Conference, 2013, pp. 375-386, doi: 10. $1145 / 2486001.2486033$.

[19] A. Goldsmith, Wireless Communications. New York, NY, USA: Cambridge University Press, 2005, doi: https://doi.org/10.1017/ CBO9780511841224.

[20] D. Tse and P. Viswanath, Fundamentals of Wireless Communication. Cambridge University Press, 2005, doi: https://doi.org/10.1017/ CBO9780511807213.

[21] C. Fragouli and E. Soljanin, "Network coding applications," Foundations and Trends in Networking, vol. 2, no. 2, pp. 135-269, 2008, doi: 10.1561/1300000013. [Online]. Available: http://dx.doi.org/10.1561/ 1300000013

[22] M. C. de Almeida Castro, "Taxas alcancáveis e projeto de códigos para o canal de retransmissão bidirecional," Ph.D. dissertation, Universidade Federal de Santa Catarina, 2016. [Online]. Available: $\langle$ http://www.bu.ufsc.br/teses/PEEL1703-T.pdf

[23] J. B. Kenney, "Dedicated short-range communications (dsrc) standards in the united states," Proceedings of the IEEE, vol. 99, no. 7, pp. 11621182, July 2011, doi: 10.1109/JPROC.2011.2132790.

[24] IEEE, "Part 11: Wireless lan medium access control (mac) and physical layer (phy) specifications," IEEE Std 802.11-2012, IEEE Standards Association, Tech. Rep., 2012, doi: 10.1109/IEEESTD.2012.6178212.

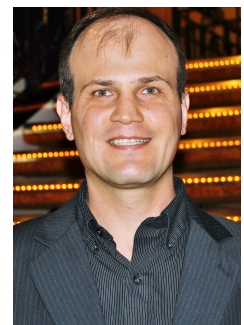

Marcio Henrique Doniak was born in Florianópolis - SC, Brazil, in 1979. After enjoying the RWTHAachen University, in Germany, for a one-year internship in September 2001, he got the B.Sc. and M.Sc. degrees from Federal University of Santa Catarina (UFSC), Florianópolis, in 2003 and 2006. From 2003 to 2004, he worked at the P\&D of the Certi Foundation with image processing. Later, from 2004 to 2008 worked with signal processing for cordless phone development and PBX at Intelbras' $P \& D$, and since 2009 he is professor of the Telecommunications Area at Federal Institute of Santa Catarina (IFSC). As a doctoral student of Electrical Engineering at UFSC, his research interests involves wireless communication and signal processing.

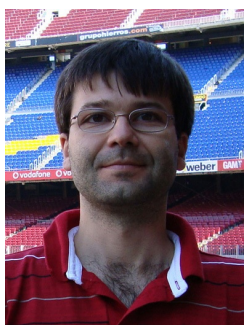

Richard Demo Souza was born in FlorianópolisSC, Brazil. He received the B.Sc. and the D.Sc. degrees in Electrical Engineering from the Federal University of Santa Catarina (UFSC), Brazil, in 1999 and 2003, respectively. In 2003 he was a Visiting Researcher in the Department of Electrical and Computer Engineering at the University of Delaware, USA. From 2004 to 2016 he was with the Federal University of Technology Paraná (UTFPR), Brazil. Since 2017 he has been with the Federal University of Santa Catarina (UFSC), Brazil, where he is an Associate Professor. His research interests are in the areas of wireless communications and signal processing. He is a Senior Member of the IEEE and of the Brazilian Telecommunications Society ( $\mathrm{SBrT}$ ), and has served as Editor-in-Chief of the SBrT Journal of Communication and Information Systems; and Associate Editor for the IEEE Communications Letters, the EURASIP Journal on Wireless Communications and Networking, and the IEEE Transactions on Vehicular Technology. He is a co-recipient of the 2014 IEEE/IFIP Wireless Days Conference Best Paper Award, the supervisor of the awarded Best PhD Thesis in Electrical Engineering in Brazil in 2014, and a co-recipient of the 2016 Research Award from the Cuban Academy of Sciences. 


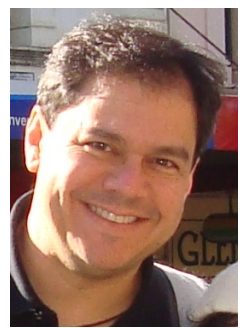

Bartolomeu F. Uchôa-Filho (S'94 - M'96 - SM'12) was born in Recife, Brazil, in 1965. He received the B.S.E.E. degree from the Federal University of Pernambuco (UFPE), Recife, Brazil, in 1989; the M.S.E.E. degree from the State University of Campinas (UNICAMP), Campinas, Brazil, in 1992; and the Ph.D. degree in electrical engineering from the University of Notre Dame, Notre Dame, Indiana, U.S.A., in 1996.

During 1997-1999, he held a Postdoctoral position at the State University of Campinas. From August 1999 to January 2000 he was a Visiting Researcher in the Department of Electrical Engineering, Federal University of Santa Catarina, Florianópolis, Brazil. Since February 2000 he has been with the same Department, where he is a Full Professor. From March 2009 to February 2010, he was a PostDoctoral Fellow at the University of Sydney, Australia. From April to June 2017, he had a joint appointment as Senior Research Fellow in the Laboratoire des Signaux et Systèmes (L2S), Centrale-Supélec, and in the Laboratoire de Traitement du Signal et Architectures Électroniques (LAETITIA), Conservatoire National des Arts et Métiers (CNAM), both in France. His research interests are in the area of coding and information theory, with applications to digital communications systems.

Dr. Uchôa-Filho is an IEEE Senior Member, a Senior Member of the Brazilian Telecommunications Society (SBrT), a member of Eta Kappa Nu, the IEEE Information Theory Society, the IEEE Communications Society, and the ARC Communications Research Network (ACoRN, Australia). For the 2003-2004 period, he served as the Editor-in-Chief for the Journal of the Brazilian Telecommunications Society. During 2011-2014, he was an Associate Editor of Information and Coding Theory for the Journal of Communication and Information Systems (JCIS). Since 2016 he has been an Associate Editor of Physical Communication (Elsevier) and Digital Signal Processing (Elsevier). He was the Technical Program Committee Co-Chair of the 27th Brazilian Telecommunications Symposium (SBrT'09), the TrackChair of the Information Theory and Coding Track of the 2014 IEEE/SBrT International Telecommunications Symposium (ITS'14), and has also served as TPC member of several national and international symposia. He is a Research Productivity Fellow (Level 1C) of the CNPq (the Brazilian National Council for Scientific and Technological Development, Ministry of Science and Technology). 